\title{
Smart monitoring information system based on RF 433 Mhz (SMIS)
}

\author{
Hicham Ouldzira ${ }^{1}$, Ahmed Mouhsen ${ }^{2}$, Hajar Lagraini $^{3}$, Abdelmoumen Tabyaoui ${ }^{4}$ Mostafa Chhiba $^{5}$ \\ ${ }^{1,2}$ Laboratory of Mechanical, Engineering, Industrial Management and Innovation, \\ The Faculty of Sciences and Technology, Hassan 1st University, Morocco \\ ${ }^{3,4,5}$ Radiation Materials and Instrumentations Laboratory, The Faculty of Sciences and Technology, \\ Hassan 1st University, Morocco
}

\begin{tabular}{|c|c|}
\hline Article Info & ABSTRACT \\
\hline Article history: & Many research works are devoted to the design of remote control systems of \\
\hline Received Apr 4, 2019 & objects. Remote monitoring is among the technologies of data acquisition on \\
\hline Revised Jul 18, 2016 & These data can be collected by acoustic waves, the distribution of forces and \\
\hline Accepted Jul 26, 2016 & $\begin{array}{l}\text { electromagnetic energy to process them to locate the object and its } \\
\text { characteristics. This paper presents a smart monitoring information system }\end{array}$ \\
\hline ovwords. & \\
\hline
\end{tabular}

Arduino nano and UNO

IoT

Remote monitoring

RF433MHZ

WSN

Copyright $₫ 2019$ Institute of Advanced Engineering and Science. All rights reserved.

Corresponding Author:

Hicham Ouldzira,

Laboratory of Mechanical, Engineering,

Industrial Management and Innovation,

The Faculty of Sciences and Technology,

Hassan 1st University,

PO Box 577, Settat, Morocco.

Email: odzira@yahoo.fr

\section{INTRODUCTION}

The progressive development of the Internet of Things (IoT) [1] provides a smart environment that helps people in their daily lives. Deployment of dense and interconnected wireless sensor networks (WSNs) is critical to IoT implementation [2]. Object monitoring based primarily on wireless sensor networks [3]. Currently, its value has increased significantly in different areas, mainly in the control of rare and expensive items. Wireless communication [4] is a transfer of information over a distance wirelessly. This distance can be short [5] (a few meters) or long (thousands or millions of kilometers for radio communications). There are different technologies that are used in wireless communications and subsequently object monitoring, such as GSM [6], Wi-Fi [7], Bluetooth [8], ZigBee [9], 6loWPAN [10], etc.The monitoring system studied essentially requires basic modules, such as microcontroller, RF modules, and GSM module.

To implement the object in a monitoring field, it is necessary to identify the range between the transmitter and the receiver RF433MHZ, so that a function between the length of the antenna RF433MHZ and this distance is required. We will then attach a remote monitoring system to the object to control it in real time [11]. This system is based on an Arduino Nano, an RF433MHZ module, a GSM module and a BUZZER module. The rest of this paper is organized as follows: 
In section 2, we will discuss the related works, in section 3 we present our proposed system SMIS, a Wireless Sensor And Actuator Network are presented in section 4, the experimental results of the function between the length of the RF 433MHZ module antenna and the distance between its receiver and its transmitter at the laboratory scale is discussed in section 5, Design and Implementation are presented in section 6 , the paper will be conclude in section 7 .

\section{RELATED WORK}

Healthcare area in recent years has been rapidly integrating technology in the monitoring, diagnosis and treatment of patients remotely. To improve quality of life of patient and to facilitates tracking of patient who needs real time data collection. Most studies reviewed point to a chronic disease monitoring in particular as in which are responsible for the first remote monitoring of vital signs and the second of a telemedical ECG system of a patient [12].

Other systems such as those proposed are fixed in the IoT bring advantages in terms of perception, transmission and application of information in the field perspectives of health and medical care. Enabling smart, an accessible and communication system based on IoT hosting segments such as: medical equipment, information management control medication of patients, telemedicine, mobile medical care, and personal health management, among others.

Home automation or Smart Homes (also known as domotic) can be described as introduction of technology within the home environment to provide convenience, comfort, security and energy efficiency to its occupants [13]. Adding intelligence to home environment can provide increased quality of life for the old-people and disabled people who might otherwise need caregivers or institutional care.

There hase been a significant gruth in home automation lastely due to higher affordability and progression in Smart phones and tablets which permits huge and easy connectivity. With the introduction of the IoT, the studies and employment of smart homes are getting more popular [6]. Much of the research attention has been given in academic research laboratories. Several wireless communication technologies that can support some form of distant data transmission, detecting and control such as Bluetooth, Wi-Fi, RFID, and cellular networks have been used to insert numerous stages of intelligence in the household [14]. Researches in [13, 14] have intreduced Bluetooth based home automation systems using Smart phones without the Internet controllability. The appliances are physically connected to a Bluetooth sub-controller which is then accessed and controlled by the Smart phone utilizing built-in Bluetooth connectivity. However, due to limited distance of operation (up to $100 \mathrm{~m}$ only) the network is incapable to manage with mobility and can only be controlled within the vicinity. Academics have also tried to provide network interoperability and distant access to control appliances at the house based on home-gateways. [17] Presented a Wi-Fi using home control system based on web server which accomplishes the connected home appliances.

A GSM based communication and control for home devices has also been introduced by [18] where diverse AT commands are transmited to the House Mobile for controlling diverse devices. The disadvantage of this network is that manipulators are not provided with a graphical user interface and they have to remember diverse AT commands to control the attached appliances.

The above cited systems have made momentous contributions to the design and improvement of domotic systems. Though, the current works were generally focused on switching and controlling home devices or connected appliances rather than distantly monitoring of home environment.

\section{THE PROPOSED SYSTEM: SMIS}

This section describes the proposed architecture and design of flexible and low cost controlling and monitoring system. The architecture is divided into three layers: monitoring area, communication module and Remote Environment (smartphone, tablet). Remote Environment represents authorized users who can visualize the sensed data on their Smart phone using sms via GSM. Monitoring district consists of a software and a hardware interface module as demonstrated in the Figure 1 below.

The diagram of the control system of Figure 1 can be divided into two parts:

a. The top part includes a GSM module, tablets, smart phones, etc.A tablet or smartphone serves as a remote control via GSM.

b. The lower part includes an intelligent central controller, RF modules, control modules, environmental modules, data collectors, and so on. The intelligent central controller is a link between the previous and the next in that it combines the top and the bottom. He is responsible for setting up a WSAN RF433 MHz (Wirless Sensors \& Actors Network) in a studied envirement. These versatile control modules are responsible for controlling the devices and communicating with the central controller. 
The smart control system is effective due to the following advantages:

- It maintains a dynamic balance through self-configuration and self-organization.

- It is easy to set up and maintain.

- The control modules have been designed in standard sizes.

The controller and these modules constitute a WSAN.

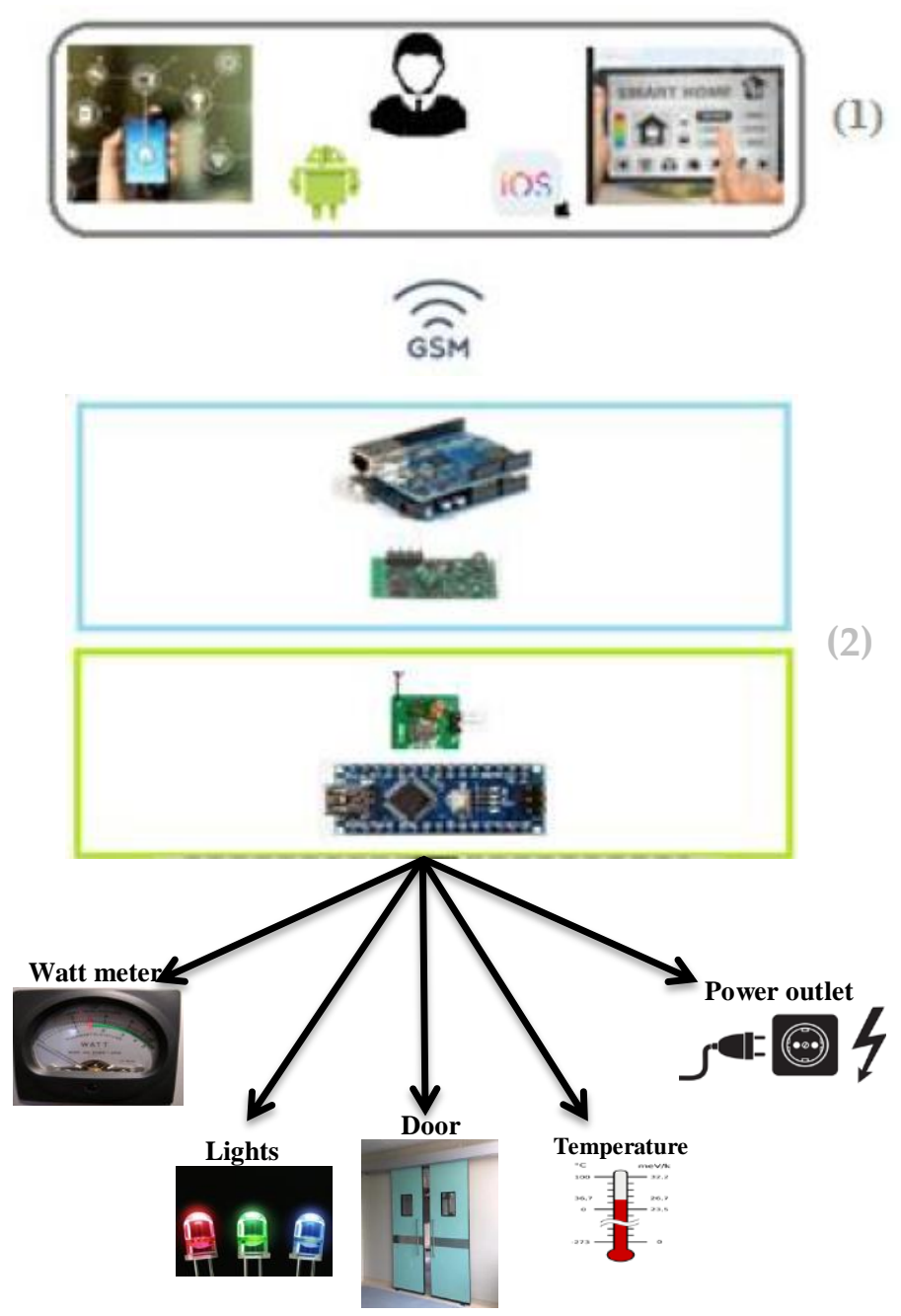

Figure 1. The proposed remote monitoring system

\section{A WIRELESS SENSOR AND ACTUATOR NETWORK}

Wireless communications namely Zigbee, Wi-Fi, Bluetooth and $433 \mathrm{MHz}$, have been widely used in many applications recently. the following Table 1 gives a comparison between these wireless technologies in terms of band operated, energy consumption, range, debit and battery life [19]:

Table 1. Comparison between these wireless technologies

\begin{tabular}{cccccc}
\hline Wireless Technologies & Band perated & Energy consumption & Transmission distance & Battery life & debit \\
\hline Zigbee & $2.4 \mathrm{GHz}$ & high & Small to medium & few days & $<1 \mathrm{Mbps}$ \\
Wi-Fi & $2.4 \mathrm{GHz}$ & high & Small to medium & few days & $1->54 \mathrm{Mbps}$ \\
RF & $433 \mathrm{MHz}$ & low & medium to large & up to 10 years & $9.6 \mathrm{Kbps}$ \\
\hline
\end{tabular}

$433 \mathrm{MHz}$ wireless communication is available worldwide and an open source alternative. It is ideal for wireless sensor network applications because it can transmit / receive over very long distances without requiring high power consumption on a battery. The low input current of typical label configurations can power button cell batteries or thin-film batteries up to 10 years. 
However, the $433 \mathrm{MHz}$ bit rate is only $9.6 \mathrm{kbps}$, making it ideal for applications in which only a small amount of data needs to be transmitted. Therefore, it is generally used for modern control systems. Our WSAN used RF433 MHz wireless communication. It adopts a star topology in which the microcontroller forms the main part and is responsible for building the entire $433 \mathrm{MHz}$ wireless network. Flowcharts Figure2, Figure3 bellow describes operation steps of the proposed system:

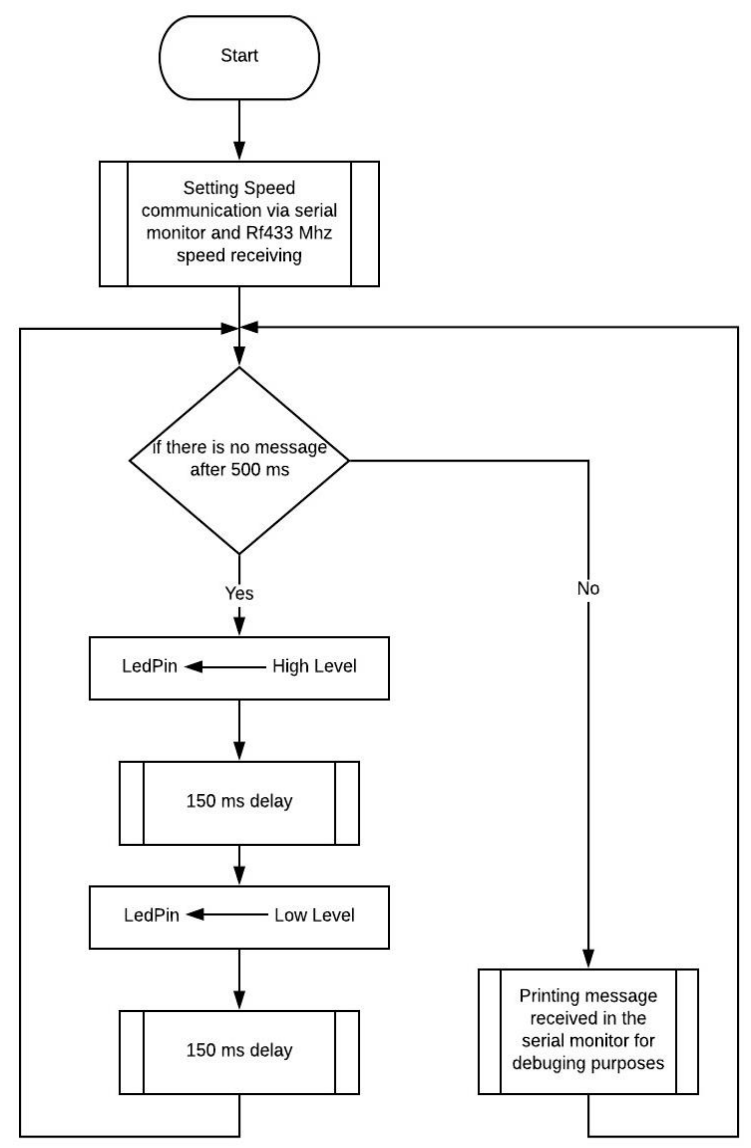

Figure 2. Operation steps of the proposed system

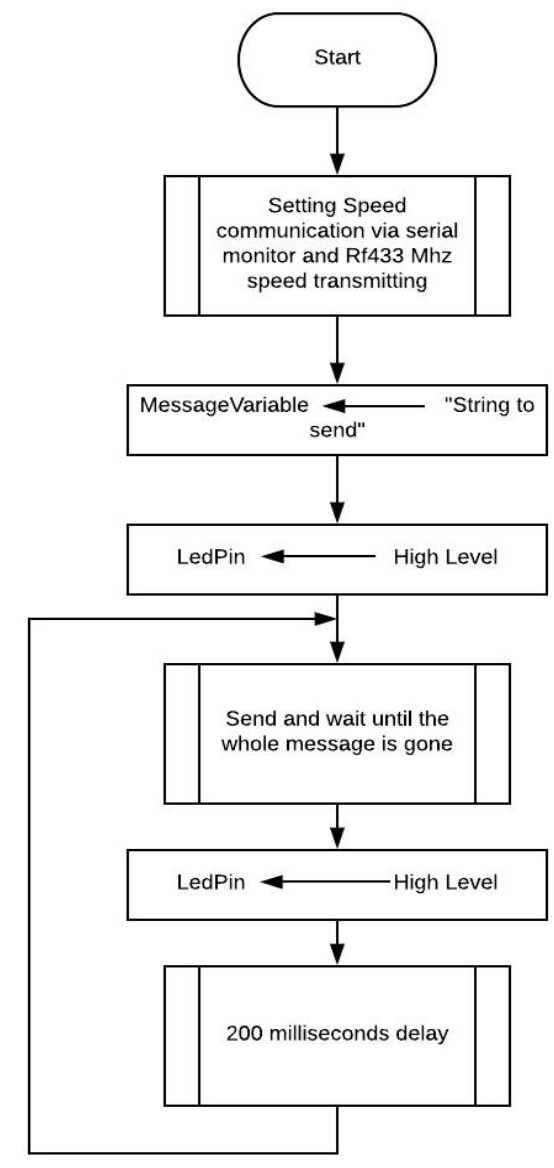

Figure 3. Operation steps of the proposed system

\section{EXPERIMENTAL RESULTS OF THE FUNCTION}

Linear model Poly9:

$$
f(x)=\sum_{n=1}^{10} p_{n} * x^{10-n}
$$

where $\mathrm{x}$ is normalized by mean 1.36 and std 0.8332

Coefficients (with $95 \%$ confidence bounds):

$\mathrm{p} 1=0.1216(-0.004683,0.2478)$

$\mathrm{p} 2=-0.2144(-0.4598,0.03102)$

p3 $=-0.3513(-1.035,0.3319)$

p4 $=0.9463(-0.2019,2.095)$

p5 $=-0.2711(-1.68,1.138)$

p6 $=-1.502(-3.206,0.2034)$

p7 $=1.415(0.177,2.652)$

$\mathrm{p} 8=1.635(0.7719,2.499)$

p9 $=2.029(1.679,2.38)$

$\mathrm{p} 10=3.775(3.668,3.883)$ 
The (1) is given by interpolation with the Newton method, and presents the following correlation performance reaches $99.94 \%$, the following table gives a summary of these performances:

Table 2. Goodness of fit

\begin{tabular}{cccc}
\hline \multicolumn{4}{c}{ Table } \\
\hline SSE & R-square & Adjusted R-square & RMSE \\
\hline 0.166 & 0.9994 & 0.9991 & 0.1052 \\
\hline
\end{tabular}

Where SSE is Sum squared error performance function, R-square is the square of the correlation between the response values and the predicted response values. It is also called the square of the multiple correlation coefficient and the coefficient of multiple determination, RMSE (Root Mean Squared Error) is known as the fit standard error and the standard error of the regression.

The measures that have been taken on a wire alloy copper $2 \mathrm{~mm}$ diameter for the RF module depending on the transmission distance.

Table 3. This table presents measurements of RF's range and antenna's length

\begin{tabular}{ccc}
\hline Number of measurestaken & Antennalength $(\mathrm{cm})$ & RF433MHz Range $(\mathrm{m})$ \\
\hline 25 & 0,1 & 0,67 \\
24 & 0,2 & 1 \\
23 & 0,3 & 1,29 \\
22 & 0,4 & 1,55 \\
21 & 0,5 & 1,61 \\
20 & 0,6 & 1,9 \\
19 & 0,7 & 2,18 \\
18 & 0,8 & 2,48 \\
17 & 0,9 & 2,77 \\
16 & 1 & 3,11 \\
15 & 1,1 & 3,33 \\
14 & 1,2 & 3,58 \\
13 & 1,3 & 3,65 \\
12 & 1,4 & 3,72 \\
11 & 1,5 & 4,06 \\
10 & 1,6 & 4,47 \\
9 & 1,7 & 4,96 \\
8 & 1,8 & 5,5 \\
7 & 1,9 & 5,98 \\
6 & 2 & 6,49 \\
5 & 2,2 & 7,55 \\
4 & 2,4 & 8,61 \\
3 & 2,6 & 9,66 \\
2 & 2,8 & 10,53 \\
1 & 3 & 14,78 \\
\hline
\end{tabular}

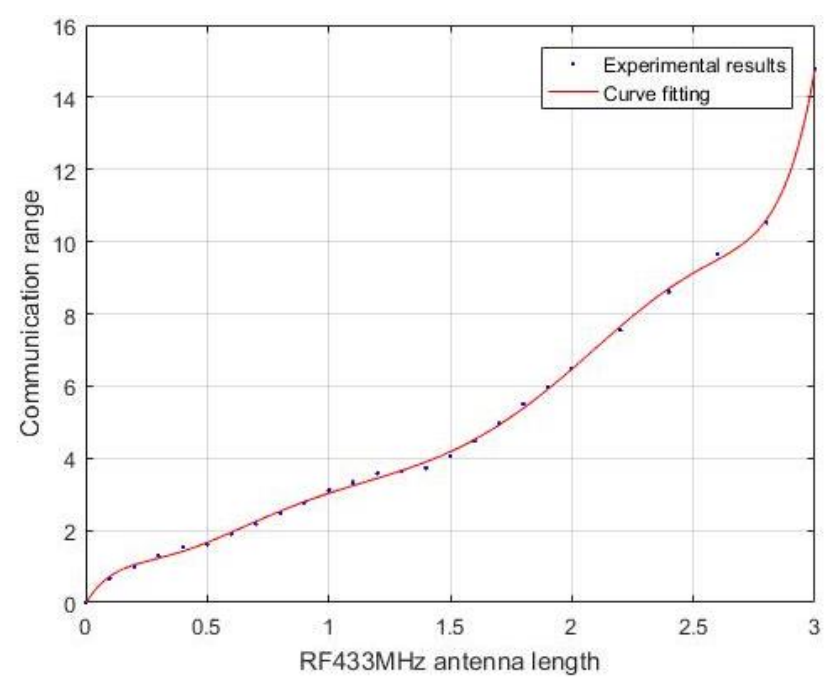

Figure 4. Communication range vs RF433MHZ antenna lentgh 


\section{DESIGN AND IMPLEMENTATION}

To manage the monitored objects remotely in specific studied area (smart home, health care, smart grid ...), it is necessary to develop a smart monitoring information system (SMIS), which can be run in a management system or on the owner's computer. We designed and developed a SMIS with arduino, smart sensors and RF433 MHZ technologies. The SMIS includes RF emitter, RF receiver, data gathering unit (Arduino Uno) and controlled objects (attached to arduino Nano), transmission module (GSM).

SMIS operates in real time, at every $500 \mathrm{~ms}$ the emitter starts the mission by sending a message to the RF 433MHZ receiver, since the remotely monitored object is included in the monitored area. If the receiver RF doesn't get any messages from the emitter, the SMIS send an SMS to the object's owner over the GMS module and activates the buzzer to tell that the object is out of the monitored area.

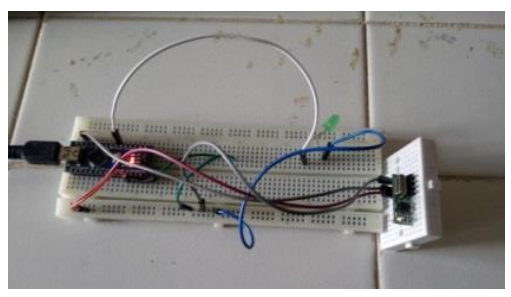

(1)

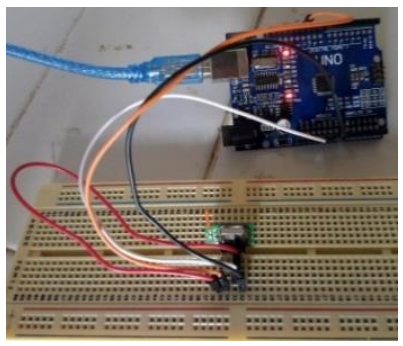

(2)

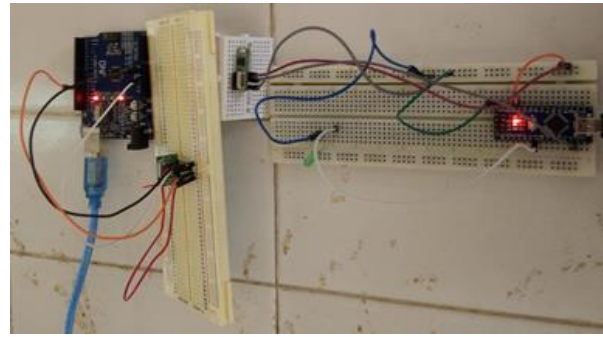

(3)

Figure 5. (1),(2) and (3) are SMIS implementation

\section{CONCLUSION}

The IoT-based object control system proposed in this document adopts the $433 \mathrm{MHz}$ wireless sensor and actuator network as a low layer, which facilitates remarkably reconfiguration and reorganization. An object owner can implement its monitoring, control and management on site or remotely by a smart terminal with GSM. Since the object control system combines a $433 \mathrm{MHz}$ wireless sensor and actuator communication network with the Internet, it can be used for power grid control, environmental monitoring, or intelligent transportation without requiring major material modification.

Enhancing energy efficiency of the actuators can reduce energy costs and emissions. After the construction of an object control system, an essential goal of the future is to manage all actuators and optimize their operation. Our current mission is to study optimal control strategies for applications that save energy in actuators.

\section{ACKNOWLEDGEMENTS}

The authors are very much thankful to the unanimous reviewers of the paper and editors of the journal for their constructive and helpful comments that improved the quality of the paper.

\section{REFERENCES}

[1] C. Kim, Y. Kim, and H. Jung, "IoT task management system using control board," Indonesian Journal of Electrical Engineering and Computer Science, vol. 13, no. 1, pp. 155, Jan. 2019.

[2] T. Taris, H. Kraimia, D. Belot, and Y. Deval, "An FSK and OOK Compatible RF Demodulator for Wake Up Receivers," Journal of Low Power Electronics and Applications, vol. 5, no. 4, pp. 274-290, Nov. 2015.

[3] Hicham Ouldzira et al, "MG-Leach: An Enhanced Leach protocol for Wireless Sensor Network," International Journal of Electrical and Computer Engineering (IJECE), vol. 9, no. 4, pp. 3139-3145, Aug 2019.

[4] B. Zhou, X.-Y.Bai, D.-H.Wang, W.-D.Wu, Y.-P.Peng, and Y. Zhang, "Intelligent Monitoring System of Pu'er Tea Warehouse Based on IoT," in Proceedings of the International Conference on Information Technology and Electrical Engineering 2018 - ICITEE '18, Xiamen, Fujian, China, 2018, pp. 1-4.

[5] H. OULDZIRA and al., "Remote monitoring of an object using a wireless sensor network based on NODEMCU ESP8266," Indonesian Journal of Electrical Engineering and Computer Science (IJEECS), Dec-2019.

[6] R. He et al., "High-Speed Railway Communications: From GSM-R to LTE-R," IEEE Vehicular Technology Magazine, vol. 11, no. 3, pp. 49-58, Sep. 2016.

[7] X. Wang, C. Yang, and S. Mao, "PhaseBeat: Exploiting CSI Phase Data for Vital Sign Monitoring with Commodity WiFi Devices," in 2017 IEEE 37th International Conference on Distributed Computing Systems (ICDCS), Atlanta, GA, USA, 2017, pp. 1230-1239. 
[8] P. Kakria, N. K. Tripathi, and P. Kitipawang, "A Real-Time Health Monitoring System for Remote Cardiac Patients Using Smartphone and Wearable Sensors," International Journal of Telemedicine and Applications, vol. 2015, pp. 1-11, 2015.

[9] A. R. Al-Ali, M. Qasaimeh, M. Al-Mardini, S. Radder, and I. A. Zualkernan, "ZigBee-based irrigation system for home gardens," in 2015 International Conference on Communications, Signal Processing, and their Applications (ICCSPA'15), Sharjah, 2015, pp. 1-5.

[10] H. Dagaleet al., "CyPhyS+: A Reliable and Managed Cyber-Physical System for Old-Age Home Healthcare over a 6LoWPAN Using Wearable Motes," in 2015 IEEE International Conference on Services Computing, New York City, NY, USA, 2015, pp. 309-316.

[11] Lu Lei, "Design of tea storage monitoring system based on wireless sensor network," Wuhan, 2016.

[12] PrashantPatil et al, "Patient Health Monitoring System using IoT," International Research Journal of Engineering and Technology (IRJET), pp. 638-641, 2017.

[13] Rajeev Piyare, "Internet of Things: Ubiquitous Home Control and Monitoring System using Android based Smart Phone," International Journal of Internet of Things, pp. 5-11, 2013.

[14] M. A. and J. S., "Integrated Wireless Technologies for Smart Homes Applications," in Smart Home Systems, M. A., Ed. InTech, 2010.

[15] R. Piyare and M. Tazil, "Bluetooth based home automation system using cell phone," in 2011 IEEE 15th International Symposium on Consumer Electronics (ISCE), Singapore, Singapore, 2011, pp. 192-195.

[16] R. A. Ramlee, D. H. Z. Tang, and M. M. Ismail, "Smart home system for Disabled People via Wireless Bluetooth," in 2012 International Conference on System Engineering and Technology (ICSET), Bandung, West Java, Indonesia, 2012, pp. 1-4.

[17] NehaAggarwal et al, "Design And Implementation Of A Wifi Based Home Automation System," World Academy of Science, Engineering and Technology, pp. 2177-2180, 2012.

[18] Md. T. Ahammed and P. P. Banik, "Home appliances control using mobile phone," in 2015 International Conference on Advances in Electrical Engineering (ICAEE), Dhaka, 2015, pp. 251-254.

[19] D. Prasad, N. N. Chiplunkar, and K. P. Nayak, "A Trusted Ubiquitous Healthcare Monitoring System for Hospital Environment:," International Journal of Mobile Computing and Multimedia Communications, vol. 8, no. 2, pp. 14-26, Apr. 2017. 